\title{
Designing of a Fleet-Leader Program for Carbon Composite Overwrapped Pressure Vessels
}

Pappu L.N. Murthy

Glenn Research Center, Cleveland, Ohio

\section{S. Leigh Phoenix}

Cornell University, Ithaca, New York 


\section{NASA STI Program . . . in Profile}

Since its founding, NASA has been dedicated to the advancement of aeronautics and space science. The NASA Scientific and Technical Information (STI) program plays a key part in helping NASA maintain this important role.

The NASA STI Program operates under the auspices of the Agency Chief Information Officer. It collects, organizes, provides for archiving, and disseminates NASA's STI. The NASA STI program provides access to the NASA Aeronautics and Space Database and its public interface, the NASA Technical Reports Server, thus providing one of the largest collections of aeronautical and space science STI in the world. Results are published in both non-NASA channels and by NASA in the NASA STI Report Series, which includes the following report types:

- TECHNICAL PUBLICATION. Reports of completed research or a major significant phase of research that present the results of NASA programs and include extensive data or theoretical analysis. Includes compilations of significant scientific and technical data and information deemed to be of continuing reference value. NASA counterpart of peer-reviewed formal professional papers but has less stringent limitations on manuscript length and extent of graphic presentations.

- TECHNICAL MEMORANDUM. Scientific and technical findings that are preliminary or of specialized interest, e.g., quick release reports, working papers, and bibliographies that contain minimal annotation. Does not contain extensive analysis.

- CONTRACTOR REPORT. Scientific and technical findings by NASA-sponsored contractors and grantees.
- CONFERENCE PUBLICATION. Collected papers from scientific and technical conferences, symposia, seminars, or other meetings sponsored or cosponsored by NASA.

- SPECIAL PUBLICATION. Scientific, technical, or historical information from NASA programs, projects, and missions, often concerned with subjects having substantial public interest.

- TECHNICAL TRANSLATION. Englishlanguage translations of foreign scientific and technical material pertinent to NASA's mission.

Specialized services also include creating custom thesauri, building customized databases, organizing and publishing research results.

For more information about the NASA STI program, see the following:

- Access the NASA STI program home page at http://www.sti.nasa.gov

- E-mail your question via the Internet to help@ sti.nasa.gov

- Fax your question to the NASA STI Help Desk at $443-757-5803$

- Telephone the NASA STI Help Desk at 443-757-5802

- Write to: NASA Center for AeroSpace Information (CASI) 7115 Standard Drive Hanover, MD 21076-1320 


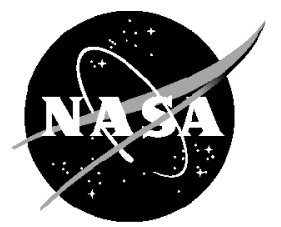

\section{Designing of a Fleet-Leader Program for Carbon Composite Overwrapped Pressure Vessels}

\section{Pappu L.N. Murthy}

Glenn Research Center, Cleveland, Ohio

\section{S. Leigh Phoenix}

Cornell University, Ithaca, New York

Prepared for the

50th Structures, Structural Dynamics, and Materials Conference

sponsored by AIAA, ASME, ASCE, AHS, and ASC

Palm Springs, California, May 4-7, 2009

National Aeronautics and

Space Administration

Glenn Research Center Cleveland, Ohio 44135 


\section{Acknowledgments}

The authors wish to acknowledge the sponsorship provided by the NASA Engineering Safety Center (NESC) for the Kevlar and Carbon Independent Technical Assessment as well as supporting the NESC Composite Pressure Vessel Working Group.

This report contains preliminary findings, subject to revision as analysis proceeds.

Level of Review: This material has been technically reviewed by technical management.

Available from

NASA Center for Aerospace Information 7115 Standard Drive

Hanover, MD 21076-1320
National Technical Information Service 5285 Port Royal Road Springfield, VA 22161

Available electronically at http://gltrs.grc.nasa.gov 


\title{
Designing of a Fleet-Leader Program for Carbon Composite Overwrapped Pressure Vessels
}

\author{
Pappu L.N. Murthy \\ National Aeronautics and Space Administration \\ Glenn Research Center \\ Cleveland, Ohio 44135 \\ S. Leigh Phoenix \\ Cornell University \\ Ithaca, New York 14853
}

\begin{abstract}
Composite Overwrapped Pressure Vessels (COPVs) are often used for storing pressurant gases on board spacecraft when mass saving is a prime requirement. Substantial weight savings can be achieved compared to all metallic pressure vessels. For example, on the space shuttle, replacement of all metallic pressure vessels with Kevlar COPVs resulted in a weight savings of about 30 percent. Mass critical space applications such as the Ares and Orion vehicles are currently being planned to use as many COPVs as possible in place of all-metallic pressure vessels to minimize the overall mass of the vehicle. Due to the fact that overwraps are subjected to sustained loads during long periods of a mission, stress rupture failure is a major concern. It is, therefore, important to ascertain the reliability of these vessels by analysis, since it is practically impossible to show by experimental testing the reliability of flight quality vessels. Also, it is a common practice to set aside flight quality vessels as "fleet leaders" in a test program where these vessels are subjected to slightly accelerated operating conditions so that they lead the actual flight vessels both in time and load. The intention of fleet leaders is to provide advanced warning if there is a serious design flaw in the vessels so that a major disaster in the flight vessels can be averted with advance warning. On the other hand, the accelerating conditions must be not so severe as to be prone to false alarms. The primary focus of the present paper is to provide an analytical basis for designing a viable fleet leader program for carbon COPVs. The analysis is based on a stress rupture behavior model incorporating Weibull statistics and power-law sensitivity of life to fiber stress level.
\end{abstract}

\section{Nomenclature}

\section{Symbols}

$F \quad$ lifetime distribution function

$P_{f} \quad$ probability of failure

$R \quad$ reliability

$S \quad$ stress ratio

$N \quad$ number of vessels

$\sigma \quad$ fiber stress

$\sigma_{\text {ref }} \quad$ fiber stress at burst pressure

$t \quad$ time in hours

$t_{d} \quad$ design life

$t_{\text {ref }} \quad$ characteristic time corresponding to $\sigma_{\text {ref }}$

$\rho \quad$ power-law coefficient for stress

$\beta \quad$ lifetime shape parameter

$\alpha \quad$ Weibull shape parameter for fiber strength

$\theta$ power law exponent to represent damage level 


\section{Subscripts}

$f l, O p$, op fleet leaders, operational vessels and operating condition

$d, D$, crit design, degraded and critical condition

\section{Introduction}

Composite Overwrapped Pressure Vessels (COPVs) are often used for storing pressurant gases onboard spacecraft. Kevlar, glass, carbon and other more recent fibers have all been used as overwraps. COPVs with metal liners are susceptible to many of the same failure modes as metallic pressure vessels, but additional considerations are required to ensure that the vessel has a reliable composite overwrap. Due to the fact that overwraps are subjected to sustained loads for an extended period during a mission, stress rupture failure is a major concern. Such failures can occur at loads that are much lower than the static strength of these vessels. A COPV that fails in stress rupture will burst suddenly without warning leading to catastrophic consequences such as loss of a vehicle and its crew.

In order to assure safe and reliable operation of such vessels, it is often a requirement to show by appropriate analyses that the failure probability due to stress rupture is low enough (i.e., the reliability is high enough) to satisfy predetermined mission requirements. There are a number of models available in the literature that can be used to estimate the reliabilities of COPVs. However, the model parameters must be determined from available test data using accepted statistical techniques such as maximum likelihood or Bayes methods. For example, for Kevlar COPVs there exists a large database of stress rupture lifetime data that was generated primarily by Lawrence Livermore National Laboratory (LLNL), Cornell University and the NASA Johnson Space Center (JSC) and with Kevlar material characterization contributions from the Y12 Plant at Oak Ridge National Laboratory and Sandia National Laboratories. These tests have involved single fibers, fiber bundles (yarns), resin impregnated strands (or tows), and laboratory scale COPVs loaded at a single constant stress level (Refs. 1 and 3 to 6) for times ranging from a few minutes to many years. Similar data for carbon vessels is largely lacking, thus forcing one to use other means of establishing stress-rupture lifetime, and mitigation procedures to reduce failure risk (Ref. 2).

In the absence of sufficient quality stress-rupture lifetime data, an alternative approach often proposed to mitigate stress rupture risk is to establish a program of so-called "fleet leader" COPVs, that is subscale vessels that lead the fleet in terms of time or fiber stress level under load and possibly under higher temperature. Such approaches have been used in the past for both Kevlar and carbon fiber COPVs. For example flight quality sub-scale Kevlar COPVs were put in a test program at JSC at slightly higher fiber load level than the actual fleet on the Orbiter before the Space Shuttle missions started. The vessels had similar wrap patterns and the same material system for the wrap as the fleet vessels on Orbiter (though recent analysis has shown that the fleet leader fiber stress ratios were actually below those for the largest vessels in the Orbiter fleet). A few of the vessels were also put under more severe accelerated conditions by subjecting them to much higher operating temperatures. For the International Space Station (ISS) program, several carbon COPVs of different sizes were impact damaged prior to placing them in a fleet leader test program. The vessels were also pressurized to slightly higher loads than the operating loads of typical ISS COPVs. The program is still ongoing after more than 9 years, and so far no failures have occurred.

A formal design/analysis procedure for setting up a fleet leader program is, however, not available. Thus the basis for setting up the number of vessels and the operating conditions for the fleet leaders has been largely 'ad hoc' and based on the particular bias of the program engineer. In the present paper a formal analytical procedure is developed, based on the classic reliability model (Refs. 1 and 7 to 10) for COPVs, by which means one can design the number of fleet leaders required and the accelerating operating conditions (pressure, time, temperature) necessary to meet the goals of a typical fleet leader program. Typically such goals are to provide legitimate advance warning when risk of failure is becoming unacceptable (due predictable material degradation but where model parameters imprecise, or due to 
overlooked errors in the manufacturing process), but at the same time not have accelerating conditions severe enough to result in false alarms.

\section{Fleet Leader Concepts}

The main purpose of a fleet leader program is to address statistical uncertainty in forecasting the reliability of a COPV, which may have been designed and manufactured according to a particular reliability model to serve for some long design lifetime, $t_{d}$. The key idea is to perform lifetime tests on several vessels that are either identical to or are accurate scale models of the vessel in service, and to use some failure rate accelerating condition such as a higher pressure, or a higher temperature or a faster time accumulation at constant load (which requires that the actual vessel in service lags the fleet leaders by spending much of its time unloaded). True fleet leaders correspond to the use of exact replicas and operating conditions of the service vessel but at a higher rate of usage. However, the term 'fleet leader' is also often used to describe scale models put under failure rate accelerating conditions, but this requires more sophistication in terms of a reliability model that can transform one scale and stress state to another.

One role of fleet leader is to expose potential errors in design, quality control or manufacturing before they lead to the catastrophic failure in fleet itself. If true, the acceleration in loading conditions should be severe enough to produce failure of a fleet leader with high probability long before the end of design life; for example the fleet leader failure probability becomes $>0.95$. This would sound a so-called 'true alarm' that vessels in service appear to have reliabilities for their intended lifetimes that are far less than originally calculated by the model, which means that some life-shortening factor was overlooked, such as use of a set of poor quality spools, or pre-preg whose shelf-life had long expired. (For instance the true vessel reliability is now only 0.995 rather than the 0.999999 or six nines it was originally designed to meet).

On the other hand, if no such design or manufacturing errors have occurred (beyond the well characterized material variability and design tolerances), then the accelerating condition should be moderate enough that the probability of failure of a fleet leader over the lifetime is appropriately low (say $<0.01$ or one-in-a-hundred) since such a failure would become a 'false alarm' that would initiate a timeconsuming, expensive and inconclusive investigation. Note that in the actual flight vessel application, the required reliability over the service life may be several nines (e.g., 0.99999 or $P_{f}=0.00001$ ). Thus the fleet leader is screening for unanticipated errors in design or manufacturing in a context where the probability of failure in service is designed to be small to begin with.

A fundamental point is that the degraded reliability level (or future reliability) implied by the failure of a fleet leader and the time of occurrence is a key consideration in setting the load or temperature parameters of the failure rate acceleration. For instance, the original design may have called for 0.999999 or six nines reliability, but once put into service, serious corrective action may not become mandatory until the reliability degrades to below 0.999 or three nines. The reason for such a double standard is that repair or replacement may itself pose risks that must be taken into account, as well as expenditure of resources that might better be spent on eliminating the sources of error or uncertainty in future replacement vessels that originally led to the degraded performance. Such decision aspects must be taken into account in the initial design of the fleet leader program and the setting of the acceleration factors; they should not be deferred until after a fleet leader has failed since the decision process will be 'ad hoc', and thus, much less likely to be objective. Conversely if the parameters of the fleet leader program are not properly set, then whether fleet leaders fail or not will have little meaning in terms of the future reliability of the vessels in service.

In order to design a service vessel to a high reliability standard one must have a robust reliability model in terms of service pressure, various design factors and stochastic material properties. This model should also form the basis for determining failure acceleration factors corresponding to desired low probability thresholds for false alarms. If the model is (1) known from long experience to accurately reflect the probability of failure of the service COPV for all relevant lifetimes, and (2) all the parameter values of the model are accurately known based on an extensive material database, then the fleet leaders 
serve mainly as confirmation of this fact, i.e., they provide some comfort in terms of a safety margin. In more technical terms, for these conditions to be true requires that all the uncertainty be "aleatory" (naturally occurring variability that is irreducible) (Refs. 11 and 12), so that the probability of failure calculations are straightforward for a given pressure level and one only needs to design to the correct service pressure to get the required reliability.

The more frequent and troublesome situation, however, is where various uncertainties exist in the vessel design and fabrication, and particularly in the properties of the overwrap materials, mainly because of lack of sufficient supporting data and the wide variety of possible material combinations as well as processing and fabrication errors that are possible. For instance, one may have very good information on how such overwrap materials behave in general, but be unclear about the behavior of this particular brand of overwrap fiber or manufacturing lot since the factory may be newly built and has an inexperienced technical staff, or it is a new relatively untested version of fiber. All this may be reflected primarily in uncertainties in the true values of the overall model parameters, which if significantly different from those assumed in the vessel design, could significantly compromise the reliability. In technical terms, this means there is considerable "epistemic" (uncertainty due to lack of knowledge or proper models or Data etc.) uncertainty (Refs. 11 and 12) to add to the previously mentioned aleatory uncertainty, making reliability predictions at a high level of confidence problematic.

\section{Fleet Leader Program Modeling Details}

Stress rupture life prediction can be accomplished using the so-called classic model as originally pioneered by Coleman (Ref. 7) and further developed by Phoenix and colleagues (Refs. 5, 6, and 8 to 10) over the past 27 years. More recently this model has undergone a thorough review during two independent technical reviews and assessments sponsored by the NASA NESC (NASA Engineering Safety Center) (Refs. 1 and 2). The model is based on a Weibull distribution framework for strength and lifetime with the embodiment of a power law to describe damage in a composite versus stress level. Derivation of the model is available in References (Refs. 7 and 8) where the power-law in stress level (with temperature dependence) is derived from thermally activated chain scission using a Morse potential as a model (Ref. 8). In the simplest setting of constant stress applied quickly and maintained over a long time period, the basic equation for the model is

$$
F\left(t, \sigma_{o p}\right)=1-\exp \left[-\left.\left\{\left.\left(\frac{t}{t_{\text {ref }}}\right)\left(\frac{\sigma_{o p}}{\sigma_{\text {ref }}}\right)^{p}\right|_{\mid} ^{\beta}\right\}\right|_{\mid}\right]
$$

where $F(t, \sigma)$ represents the probability of failure at time $t$. In the above equation the quantity $\left(\sigma_{o p} / \sigma_{\text {ref }}\right)$ is the ratio of fiber stress at operating pressure to fiber stress at burst pressure (stress ratio), $t$ is time, $t_{\text {ref }}$ is a reference time, $\rho$ is the power law exponent, and $\beta$ is the Weibull shape parameter for lifetime. The value for $\sigma_{\text {ref }}$ is determined from the flight COPV burst tests and stress analysis of the COPV. The model is shown for a single stress level over time, but for more general time histories a memory integral is used to accumulate damage (similar to Miner's rule for fatigue) at different stress levels.

In order to address issues specific to carbon fibers, an alternative model has also been proposed and developed exclusively to address carbon overwrapped vessels which are more common and fast replacing the Kevlar overwrapped vessels. The model is known as the 'fiber breakage model' the details of which are described in (Refs. 2 and 13). As this model is more complicated, it was decided to use the simple classic model to develop the analytical framework and illustrate the concepts for fleet leader design. The principles however are equally applicable to the fiber breakage model as well. 


\section{Stress Rupture Life Distribution Model}

To define a successful fleet leader program one must determine, the number of vessels, $N_{f l}$, the stress ratio at which fleet leaders must be in operation, $S_{f}$, and the probability of failure of a fleet leader due to the inherent and well-characterized natural variability (which would produce a false alarm). The classic model for the stress rupture lifetime distribution can be used to construct an analytical framework for designing a fleet leader program. As mentioned before, the probability of failure of a single fleet leader vessel up to time $t \geq 0$ is well modeled by the lifetime distribution function given in Equation (1) and rewritten as

$$
F_{1}\left(t ; \sigma, \sigma_{\text {ref }}, t_{\text {ref }}, \rho, \beta\right)=1-\exp \left\{-\left[\left.\left(\frac{\sigma}{\sigma_{\text {ref }}}\right)^{\rho}\left(\frac{t}{t_{\text {ref }}}\right)^{\mid}\right|_{\}} ^{\beta}\right\}, \quad t \geq 0\right.
$$

where the parameters are defined as follows

$\sigma_{\text {ref }}$ is a reference stress taken as the observed fiber strength at burst at some prescribed loading rate, or more precisely, the Weibull scale parameter of the 'delivered' fiber strength at burst for a single vessel;

$t_{\text {ref }} \quad$ is a characteristic time corresponding to $\sigma_{\text {ref }}$ (i.e., the projected Weibull scale parameter for lifetime at the fiber stress $\sigma=\sigma_{\text {ref }}$;

$\rho \quad$ is the power-law exponent relating lifetime to stress level (i.e., $t \propto \sigma^{-\rho}$ whereby $\log \sigma$ versus $\log$ $t$ plots as a straight line with slope $-1 / \rho$ );

$\beta \quad$ is the Weibull scale parameter observed for life times, which can be measured from stress rupture tests.

Note that in a burst test, the Weibull shape parameter for fiber stress at burst can be shown to be $\alpha ; \beta \rho$, so there is dependency among the parameters. Likewise $\sigma_{\text {ref }}$ and $t_{\text {ref }}$ are related and depend on the pressure rate used in the burst tests. This version of the model does not assume previous survival of a proof test of any kind, though the same principles apply with the appropriate conditional lifetime distribution.

We assume that $N_{o p}$ vessels are to be placed in service at operating pressure $p_{o p}$ which produces fiber stress $\sigma_{o p}$ and fiber stress ratio

$$
S_{o p}=\sigma_{o p} / \sigma_{\text {ref }}
$$

We also assume there are $N_{f l}$ fleet leaders to be put in test under controlled but accelerating conditions at fleet leader pressure $p_{f l}$ (to be determined), which produces fiber stress $\sigma_{f l}$ and fiber stress ratio

$$
S_{f l}=\sigma_{f l} / \sigma_{\text {ref }}
$$

(In this paper we shall only consider increased pressure or stress ratio as an accelerating condition.) Uncertainties in the vessel design and fabrication techniques, and in the overwrap materials are reflected primarily in uncertainties in the true values of the model parameters $\sigma_{\mathrm{ref}}, t_{\mathrm{ref}}, \rho$, and $\beta$ in that some values might be degraded to varying degrees, in which case the reliability calculation assumed in the initial design is much too optimistic. 
The two scenarios described in the beginning are formalized as follows:

(1) If the service time on the $N_{o p}$ vessels is intermittent, i.e., only a few days per year at operating pressure, $p_{o p}$, and the remaining time is spent at greatly reduced pressure with respect to stress-rupture, then the $N_{f l}$ fleet leader vessels may be pressurized to a pressure $p_{f l}$ giving the same fiber stress ratio $S_{o p}$ as in the service vessels (though if the vessels are not identical in design but are scale models, it may be that $\sigma_{f l} \neq \sigma_{o p}$ due to Weibull size effect adjustments). This is the situation that applies to the Orbiter fleet leaders at ambient conditions, and the idea is that whatever the model parameter values, a fleet leader has a high probability of failure well in advance of failure of a service vessel due to much more rapid time accumulation. Note that true fleet leaders are identical vessels.

(2) If the service time in the $N_{o p}$ vessels is continuous, then the $N_{f l}$ fleet leaders must be placed under accelerating conditions, for instance under a higher pressure giving a higher fiber stress $\sigma_{f}>\sigma_{o p}$ and higher fiber stress ratio

$$
S_{f l}>S_{o p}
$$

Here, the idea is that if the fiber stress ratios in the fleet leaders are high enough (but not too high), then a error in the materials quality control or design will result in model parameter values sufficiently different from those assumed in the design that the probability of failure of at least one fleet leader will be high and much higher that for the vessels in service despite their having material parameter values inferior to those that had been assumed in the original design.

To simplify matters and illustrate the key concepts we focus on the situation (2).

\section{Fleet Leader Design Approach}

The general parameter set is given by

$$
\rho, \sigma_{\text {ref }}, t_{\text {ref }}, \beta, \alpha=\beta \rho
$$

and we will consider two versions, the parameter values used in design, subscripted by ' $d$ ' and the degraded parameter set subscripted by ' $D$ '. Then we will have

$$
\begin{gathered}
\rho_{d}, t_{\text {ref }, d}, \beta_{d}, \alpha_{d}=\beta_{d} \rho_{d} \text { (design set) } \\
\rho_{D}, t_{\text {ref }, D}, \beta_{D}, \alpha_{D}=\beta_{D} \rho_{D} \text { (degraded set) }
\end{gathered}
$$

In Equation (8) we have a set of degraded model parameters that will cause reduced reliability due to either design errors or inferior materials in terms of variability or breakdown rate not screened out during quality control testing. For simplicity, we have not added a subscript to $\sigma_{\text {ref }}$ since this is usually determined in prototype burst testing and so forms the basis of the fiber stress ratios whether the properties are actually degraded or not.

First we derive some basic relationships in terms of the design parameters of the model. For a given reliability, $R_{d}=1-P_{f, d}$, with respect to survival of $N_{o p}$ vessels over the full design lifetime, $t_{d}$, we first find the operating stress ratio, $S_{o p}$, that will provide this reliability. For this we must solve

$$
F_{N_{o p}}\left(t_{d} ; S_{d} \sigma_{\text {ref }}, \sigma_{\text {ref }}, t_{\text {ref }, d}, \rho_{d}, \beta_{d}\right)=1-R_{d}
$$


With the aid of Equation (2), and noting that $\alpha_{d}=\beta_{d} \rho_{d}$, we obtain

$$
N_{o p}\left(S_{o p}\right)^{\alpha_{d}}\left(\frac{t_{d}}{t_{\mathrm{ref}, d}}\right)^{\beta_{d}}=-\ln R_{d}
$$

or

$$
\begin{aligned}
S_{o p} & =\left(\frac{-\ln R_{d}}{N_{o p}\left(t_{d} / t_{\mathrm{ref}, d}\right)^{\beta_{d}}}\right)^{1 / \alpha_{d}}=\left(\frac{-\ln \left(1-P_{f, d}\right)}{N_{o p}\left(t_{d} / t_{\mathrm{ref}, d}\right)^{\beta_{d}}}\right)^{1 / \alpha_{d}} \\
& \approx\left(\frac{P_{f, d}}{N_{o p}\left(t_{d} / t_{\mathrm{ref}, d}\right)^{\beta_{d}}}\right)^{1 / \alpha_{d}}, \quad 0<P_{f, d}<<1
\end{aligned}
$$

\section{Probability of a False Alarm}

A "false alarm" signifies that one of the fleet leaders failed due to natural causes. Suppose we wish to place $N_{f l}$ fleet leaders on test. It is first necessary to decided on an acceptable false alarm probability of failure, $P_{\text {false alarm, }}$ assuming that the design and materials exactly follow the parameter set, Equation (7), we assumed above. A reasonable choice might be $P_{\text {false alarm }}=0.01$, that is, one-in-a-hundred chance of a false alarm. This choice must balance such things as the cost of a false alarm in terms of triggered investigations and the likelihood a legitimate true alarm will be heeded if the vessels do in fact have degraded properties. On the other hand, if the value is set too low, the sensitivity of the fleet leaders in detecting truly degraded vessels is lowered.

Then to determine the operating stress ratio $S_{f l}$ for the fleet leaders, the following equation must be solved:

$$
F_{N_{f}}\left(t ; S_{f l} \sigma_{\text {ref }}, \sigma_{\text {ref, }} t_{\text {ref }, d}, \rho_{d}, \beta_{d}, N_{o p}\right)=P_{\text {false alarm }}
$$

Resulting in

$$
N_{f l}\left(S_{f l}\right)^{\alpha_{d}}\left(\frac{t_{d}}{t_{\mathrm{ref}, d}}\right)^{\beta_{d}}=-\ln \left(1-P_{\text {false alarm }}\right)
$$

which gives

$$
S_{f l}=\left(\frac{-\ln \left(1-P_{\text {false alarm }}\right)}{N_{f l}\left(t_{d} / t_{\text {ref }, d}\right)^{\beta_{d}}}\right)^{1 / \alpha_{d}} \approx\left(\frac{P_{\text {false alarm }}}{N_{f l}\left(t_{d} / t_{\text {ref }, d}\right)^{\beta_{d}}}\right)^{1 / \alpha_{d}}, 0<P_{\text {false alarm }}<<1
$$

\section{Reliability of Sounding a True Alarm}

A "true alarm" signifies that one of the fleet leaders failed due to serious problems in design, or material quality control. Once the operating stress ratio for fleet leaders is established, we must consider the probability of sounding a true alarm, $P_{\text {true alarm, }}$, if indeed the degraded parameter set, Equation (8), best 
characterizes the vessels thus reflecting a serious problem in the design or materials quality control. The probability of failure of a fleet leader under the degraded set of parameters is given by

$$
\begin{aligned}
F_{N_{f l}}\left(t ; S_{f l} \sigma_{\text {ref }}, \sigma_{\text {ref }}, t_{\text {ref }, D}, \rho_{D}, \beta_{D}, N_{f l}\right) & =1-\exp \left\{-N_{f l}\left[\left.\left(S_{f l}\right)^{\rho_{D}}\left(\frac{t}{t_{\text {ref }, D}}\right)_{]}^{\beta_{D}}\right|_{j} ^{\}}\right.\right. \\
& =1-\exp \left\{-\left.N_{f l}\left(S_{f l}\right)^{\alpha_{D}}\left(\frac{t}{t_{\mathrm{ref}, D}}\right)^{\beta_{D}}\right|_{\}}\right\}, t \geq 0
\end{aligned}
$$

and thus the probability of a true alarm is

$$
P_{\text {true alarm }}=1-\exp \left\{-\left.N_{f l}\left(S_{f l}\right)^{\alpha_{D}}\left(\frac{t}{t_{\text {ref }, D}}\right)^{\beta_{D}}\right|_{\}}\right\}, \quad t \geq 0
$$

The probability of a true alarm depends on the values of the degraded parameters relative to the original ones, but the problem can be viewed inversely in terms of the desired probabilities of sounding true alarms, avoiding false alarms and prescribing the level of reduced reliability to be associated with the degraded parameter values. From previous Equations (14) and (16) we have

$$
S_{f l}=\left(\frac{-\ln \left(1-P_{\text {false alarm }}\right)}{N_{f l}\left(t_{d} / t_{\text {ref }, d}\right)^{\beta_{d}}}\right)^{1 / \alpha_{d}}=\left(\frac{-\ln \left(1-P_{\text {true alarm }}\right)}{N_{f l}\left(t_{d} / t_{\text {ref }, D}\right)^{\beta_{D}}}\right)^{1 / \alpha_{D}}
$$

or

$$
\left(-\ln \left(1-P_{\text {false alarm }}\right)\right)^{1 / \alpha_{d}}=\left(t_{d} / t_{\text {ref }, d}\right)^{\frac{\rho_{D}-\rho_{d}}{\rho_{d} \rho_{D}}}\left(t_{\text {ref }, D} / t_{\text {ref }, d}\right)^{1 / \rho_{D}}\left(N_{f l}\right)^{\frac{1}{\alpha_{d}}-\frac{1}{\alpha_{D}}}\left(-\ln \left(1-P_{\text {true alarm }}\right)\right)^{1 / \alpha_{D}}
$$

or

$$
-\ln \left(1-P_{\text {true alarm }}\right)=\left(N_{f l}\right)^{1-\frac{\alpha_{D}}{\alpha_{d}}}\left(t_{d} / t_{\text {ref }, d}\right)^{\beta_{D}\left(1-\frac{\rho_{D}}{\rho_{d}}\right)}\left(t_{\text {ref }, d} / t_{\text {ref }, D}\right)^{\beta_{D}}\left(-\ln \left(1-P_{\text {false alarm }}\right)\right)^{\alpha_{D} / \alpha_{d}}
$$

However, $0<P_{\text {false alarm }} \ll<$ so that $-\ln \left(1-P_{\text {false alarm }}\right) \approx P_{\text {false alarm }}$ and thus

$$
P_{\text {true alarm }} \approx 1-\exp \left\{-\left(N_{f l}\right)^{1-\frac{\alpha_{D}}{\alpha_{d}}}\left[\left.\left(\frac{t_{d}}{t_{\text {ref }, d}}\right)^{1-\frac{\rho_{D}}{\rho_{d}}}\left(\frac{t_{\text {ref }, d}}{t_{\text {ref }, D}}\right)^{\beta_{D}}\left(P_{\text {false alarm }}\right)^{\alpha_{D} / \alpha_{d}}\right|_{\}}\right\}\right.
$$

Similarly we can calculate the degraded reliability, $R_{D}$ of the service vessel and this is 


$$
-\ln \left(R_{D}\right) \approx\left(N_{o p}\right)^{1-\frac{\alpha_{D}}{\alpha_{d}}}\left[\left(\frac{t_{d}}{t_{\mathrm{ref}, d}}\right)^{1-\frac{\rho_{D}}{\rho_{d}}}\left(\frac{t_{\mathrm{ref}, d}}{t_{\mathrm{ref}, D}}\right)^{\beta_{D}}\left(-\ln \left(R_{d}\right)\right)^{\alpha_{D} / \alpha_{d}}\right.
$$

Since $P_{f, d}=1-R_{d}$ and both $0<P_{f, D}<<1$ and $0<P_{f, D}<<1$, i.e., they are small compared to one, then we have $-\ln \left(R_{D}\right) \approx P_{f, D}$ and $\ln \left(R_{d}\right) \approx P_{f, d}$ so that

$$
P_{f, D} / N_{o p} \approx\left[\left(\frac{t_{d}}{t_{\mathrm{ref}, d}}\right)^{1-\frac{\rho_{D}}{\rho_{d}}}\left(\frac{t_{\mathrm{ref}, d}}{t_{\mathrm{ref}, D}}\right)^{\beta_{D}}\left(P_{f, d} / N_{o p}\right)^{\alpha_{D} / \alpha_{d}}\right.
$$

Taking logarithms of both sides we have

$$
\ln \left(\frac{P_{f, D}}{N_{o p}}\right) \approx \beta_{D}\left[\left(1-\frac{\rho_{D}}{\rho_{d}}\right) \ln \left(\frac{t_{d}}{t_{\mathrm{ref}, d}}\right)+\ln \left(\frac{t_{\mathrm{ref}, d}}{t_{\mathrm{ref}, D}}\right)\right]+\left(\frac{\alpha_{D}}{\alpha_{d}}\right) \ln \left(\frac{P_{f, d}}{N_{o p}}\right)
$$

\section{Critical Threshold of Degraded Reliability for Launching Corrective Action}

To design a fleet leader program we must specify a critical threshold value of $P_{f, D}$ below which the reliability cannot go without triggering corrective action. This can be defined in various ways, but a convenient way is to define

$$
P_{f, D, \text { crit }} \equiv\left(P_{f, d}\right)^{\theta_{D_{\text {crit }}}}
$$

where $0<\theta_{D_{\text {crit }}}<1$ is an exponent. Then we can consider a special family of 'critical damage' parameter values of the model subscripted as ' $D_{\text {crit' }}$, which, as a group, define threshold values

$$
\rho_{D_{\text {crit }}}, t_{\text {ref }, D_{\text {crit }}}, \beta_{D_{\text {crit }}}, \alpha_{D_{\text {crit }}}=\beta_{D_{\text {crit }}} \rho_{D_{\text {crit }}}
$$

Equations (21) to (23) can be combined to arrive at the following relation for critical set of damaged parameters

$$
\beta_{D_{\text {crit }}}\left[\left(1-\frac{\rho_{D_{\text {cit }}}}{\rho_{d}}\right) \ln \left(\frac{t_{d}}{t_{\text {ref }, d}}\right)+\ln \left(\frac{t_{\text {ref }, d}}{t_{\text {ref }, D_{\text {cit }}}}\right)\right]+\left(\frac{\alpha_{D_{\text {crit }}}}{\alpha_{d}}-\theta_{D_{\text {cit }}}\right) \ln \left(\frac{P_{f, d}}{N_{o p}}\right) \approx 0
$$

Then a worse than critical damage set would need to satisfy

$$
\beta_{D}\left[\left(1-\frac{\rho_{D}}{\rho_{d}}\right) \ln \left(\frac{t_{d}}{t_{\text {ref }, d}}\right)+\ln \left(\frac{t_{\text {ref }, d}}{t_{\text {ref }, D}}\right)\right]+\left(\frac{\alpha_{D}}{\alpha_{d}}-\theta_{D_{\text {crit }}}\right) \ln \left(\frac{P_{f, d}}{N_{o p}}\right)<0
$$


This analysis reveals that the parameters can combine in various ways to cause the same critical reliability state. Furthermore we can see that success of the fleet leader program in terms of having the capability to detect a true alarm without a false alarm also depends on the same parameters. Thus we have

$$
P_{\text {true alarm }} \approx 1-\exp \left\{-N_{f l}\left[\left.\left(\frac{t_{d}}{t_{\text {ref }, d}}\right)^{1-\frac{\rho_{D}}{\rho_{d}}}\left(\frac{t_{\text {ref }, d}}{t_{\text {ref }, D}}\right)^{\beta_{D}}\left(\frac{P_{\text {false alarm }}}{N_{f l}}\right)^{\alpha_{D} / \alpha_{d}}\right|_{\}}\right\}\right.
$$

and the probability of sounding true alarm will depend on the threshold value for the probability of a false alarm.

\section{Case Studies and Examples}

We now focus on some examples to illustrate several points.

\section{Case 1: Kevlar 49/Epoxy Fleet Leaders}

Suppose four Kevlar 49 overwrapped vessels are to be put in service, which must have a design life of 10 years, $\left(t_{d}=87,600 \mathrm{hr}\right)$ at a reliability level of $R_{d}=0.999999$ (six nines) or failure probability, $P_{f, d}=$ 0.000001 . We assume that the critical reliability for corrective action due to discovered damage or degraded parameter values is 0.999 or the probability of failure, $P_{f, D}=0.001$. Thus $\theta_{D_{\text {crit }}}=1 / 2$ since $P_{f, D}=\sqrt{P_{f, d}}$. We further assume that the original design parameters were

$$
\rho_{d}=24, t_{\mathrm{ref}, d}=1.3 \mathrm{hr}, \beta_{d}=1.67, \alpha_{d}=40
$$

The parameters chosen are typical for Kevlar flight quality vessels that are currently being used to computer reliability of orbiter COPVs. With the aid of Equations (2) and (14) one calculates the fiber stress ratio $S_{o p}$ to be 0.4299 . Finally, suppose we place $N_{f l}=10$ fleet leaders on test and choose $P_{\text {false alarm }}=$ 0.01 ; That is, we set the probability to be $1 / 100$ that at least one of the 10 fleet leaders fails when governed by the original design parameters. The required fleet leader stress ratio can be calculated using Equation (14) as $S_{f l}=0.529$.

In order for the fleet leaders to be useful, however, they must be capable of detecting a problem with the design and fabrication of the service vessel whereby the actual parameter values are degraded relative to those assumed in the design. For instance, we suppose that a key manufacturing process in the fiber or overwrap was mishandled and the true parameter values are instead

$$
\rho_{D}=19, t_{\mathrm{ref}, D}=0.95 \mathrm{hr}, \beta_{D}=1.79, \alpha_{D}=34
$$

and again $N_{o p}=4$. Then using Equation (20) we calculate,

$$
\begin{aligned}
P_{f, D} & \approx 4\left[\left(\frac{87600}{1.3}\right)^{1-\frac{19}{24}}\left(\frac{1.3}{0.95}\right)\right]_{]}^{1.79}\left(\frac{0.000001}{4}\right)^{34 / 40} \\
& =0.0011
\end{aligned}
$$


meaning the reliability under the degraded set of parameters values is $R_{D}=0.9989$.

The probability of at least one fleet leader failing, and thus sounding a true alarm, can be calculated for this degraded set of parameters using Equation (26) as

$$
P_{\text {true alarm }}=1-\exp \left\{-N_{f l}\left[\left.\left(\frac{t_{d}}{t_{\mathrm{ref}, d}}\right)^{1-\frac{\rho_{D}}{\rho_{d}}}\left(\frac{t_{\mathrm{ref}, d}}{t_{\mathrm{ref}, D}}\right)^{\beta_{D}}\left(\frac{P_{\text {false alarm }}}{N_{f l}}\right)^{\alpha_{D} / \alpha_{d}}\right|_{\}}\right\}\right.
$$

Viewed another way, the probability the set of fleet leaders fails to sound a true alarm when they should (i.e., they all survive) is 0.044 or about $1 / 23$. If one wishes to improve the probability of a true alarm then one might raise the probability of a false alarm to $P_{\text {false alarm }}=0.015=1 / 67$. Then a repeat of the above calculation gives $P_{\text {true alarm }}=0.988$, or failure to sound a true alarm now of $(1-0.988)=0.012=$ $1 / 83$. This illustrates the tradeoff in terms of having to raise the chance of a false alarm in order to raise the probability or reliability of the fleet leaders in sounding a true alarm, or in other words, the sensitivity of the fleet leaders to uncovering a serious design or material issue. One further observation is that, if the false alarm probability must be preserved at a low level, then the true alarm sensitivity must be increased by increasing the number of fleet leaders, $N_{f l}$.

An interesting aspect of this result is that a different set of damage parameters will give a somewhat different result with respect to a true alarm. For instance we consider the parameter set

$$
\rho_{D}=18, t_{\mathrm{ref}, D}=0.90 \mathrm{hr}, \beta_{D}=2.22, \alpha_{D}=40
$$

Then we calculate $P_{f, D}=0.00108$ or $R_{D}=0.9989$ and the probability of a true alarm is $P_{\text {true alarm }}=0.99998$. Here we could lower the probability of a false alarm to $P_{\text {false alarm }}=0.005=1 / 200$ and a quick calculation shows that failure of a true alarm is about the same at $1-P_{\text {true alarm }}=1 / 224$. These two values are more desirable than the values in the previous, even though the number of service and fleet leader vessels is the same.

One important role of fleet leaders is to give an early warning that the reliability of flight vessels to the end of the design life may be inadequate. In the example just considered we can calculate the probability of failure of a service vessel versus time for the design parameters and degraded parameters, and also do the same for the fleet leaders. We therefore plot

$$
\begin{gathered}
F_{4, d}\left(t ; S_{o p}\right)=1-\exp \left\{-4(0.4299)^{40}(t / 1.3)^{1.67}\right\} \\
F_{4, D}\left(t ; S_{o p}\right)=1-\exp \left\{-4(0.4299)^{40}(t / 0.90)^{2.22}\right\} \\
F_{10, d}\left(t ; S_{f l}\right)=1-\exp \left\{-10(0.5290)^{40}(t / 1.3)^{1.67}\right\}
\end{gathered}
$$

and

$$
F_{10, D}\left(t ; S_{f t}\right)=1-\exp \left\{-10(0.5290)^{40}(t / 0.90)^{2.22}\right\}
$$

Figure 1 shows the results. 


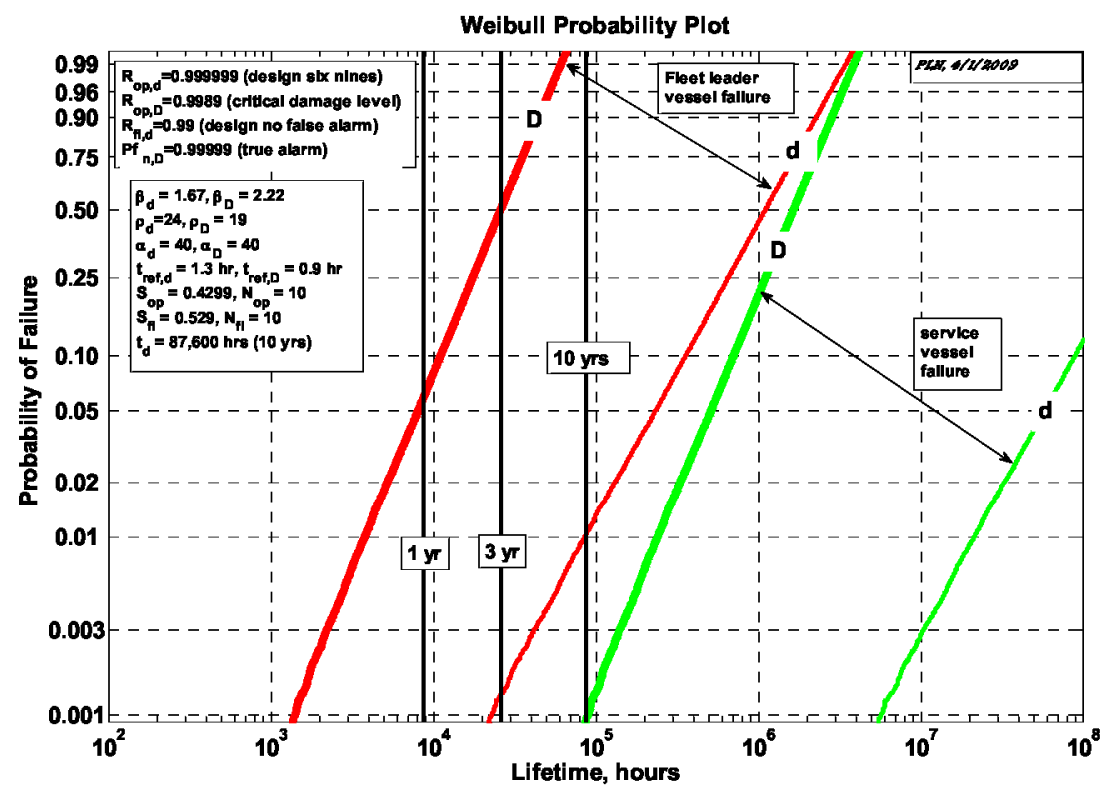

Figure 1.-Operating curves for a Kevlar COPV Fleet Leader Program to guard against possible design/manufacturing flaws and poor material quality.

One sees in Figure 1 that a Fleet leader vessel would become likely to fail and 'sound the alarm' (reach 0.5 probability of failure) by 3 years, but at one year it is only about one-in-twenty. However, the probability of failure of a service vessel is very low by then. Once again if the advance warning ( 3 years) is deemed not good enough, then one must consider raising the number of fleet leaders so that warning can be had sooner.

\section{Case 2: Carbon/Epoxy Fleet Leaders}

Carbon composite overwrapped pressure vessels are currently more popular and fast replacing the existing Kevlar vessels. They are deemed to have a far superior stress rupture life performance compared to their Kevlar counterparts. As before, suppose we have four carbon fiber/epoxy overwrapped vessels instead of Kevlar vessels that are put into service and must have a design life of 10 years, i.e., $t_{d}=87,600 \mathrm{hr}$ at a reliability level of $R_{d}=0.999999$ (six nines) or probability of failure $P_{f, d}=0.000001$. We also assume the critical reliability for corrective action due to damage to be 0.999 or the probability of failure, $P_{f, D}=0.001$. This means that $\theta_{D_{\text {crit }}}=1 / 2$ since $P_{f, D}=\sqrt{P_{f, d}}$. We assume the design parameters are

$$
\rho_{d}=120, t_{\text {ref }, d}=1.0 \mathrm{hr}, \beta_{d}=0.25, \alpha_{d}=\beta_{d} \rho_{d}=30
$$

Then the lifetime distribution Equation (1) becomes

$$
\begin{gathered}
F_{4}\left(t ; S_{o p}\right)=1-\exp \left\{-4\left[\left(S_{o p}\right)^{120}(t / 1.0)\right]^{0.25}\right\} \\
\approx 4\left(S_{o p}\right)^{30}(t / 1.0)^{0.25}, \quad t \geq 0
\end{gathered}
$$

The fiber stress ratio for this case is 


$$
S_{o p}=\left(\frac{-\ln R_{d}}{N_{o p}\left(t_{d} / t_{\mathrm{ref}, d}\right)^{\beta_{d}}}\right)^{1 / \alpha_{d}}=\left(\frac{0.000001}{4(87600)^{0.25}}\right)^{1 / 30}=0.5480
$$

Suppose we then place $N_{f l}=10$ fleet leaders on test and choose $P_{\text {false alarm }}=0.01$ that at least one of the 10 fleet leaders fails simply due to the original design being correct. The required stress ratio is

$$
S_{f l}=\left(\frac{-\ln \left(1-P_{\text {false alarm }}\right)}{N_{f l}\left(t_{d} / t_{\text {ref }, d}\right)^{\beta_{d}}}\right)^{1 / \alpha_{d}}=\left(\frac{0.01005}{10(87600)^{0.25}}\right)^{1 / 30}=0.7226
$$

In order for the fleet leader program to be useful, however, it must be capable of detecting a problem with the design/fabrication or material quality of the service vessel whereby the actual parameter values are degraded relative to those assumed in the design. For instance, we suppose that a key manufacturing process in the fiber or overwrap was mishandled, and the parameter values are instead

$$
\rho_{D}=80, t_{\mathrm{ref}, D}=1.0 \mathrm{hr}, \beta_{D}=0.225, \alpha_{D}=18
$$

and again $N_{o p}=4$. Then we calculate

$$
\begin{aligned}
P_{f, D} & \approx 4\left[\left(\frac{87600}{1.0}\right)^{1-\frac{80}{120}}\left(\frac{1}{1}\right)\right]_{]}^{0.225}\left(\frac{0.000001}{4}\right)^{18 / 30} \\
& =0.00103
\end{aligned}
$$

or reliability $R_{D}=0.9989$ for the service vessels governed by the degraded parameters, Equation (40). For the fleet leaders also with the degraded parameter set, we calculate

$$
\begin{aligned}
P_{\text {true alarm }} & =1-\exp \left\{-N_{f l}\left[\left.\left(\frac{t_{d}}{t_{\text {ref }, d}}\right)^{1-\frac{\rho_{D}}{\rho_{d}}}\left(\frac{t_{\text {ref }, d}}{t_{\text {ref }, D}}\right)^{\beta_{D}}\left(\frac{P_{\text {false alarm }}}{N_{f l}}\right)^{\alpha_{D} / \alpha_{d}}\right|_{\}}\right\}\right. \\
& =0.3097
\end{aligned}
$$

Viewed another way, the probability a fleet leader fails to sound a true alarm when it should is $1-0.3097$ $=0.6903$, which is far too high. To improve the probability of a true alarm then one must raise the probability of a false alarm. For instance if the fleet leader stress ratios is raised to $S_{f l}=0.789$ then we obtain $P_{\text {false alarm }}=0.143=1 / 7$ and $P_{\text {true alarm }}=0.841$ but this implies a probability of failing to sound a true alarm of $1-0.841=0.159 \approx 1 / 6$ which while a significant increase, is still not satisfactory.

As in the previous example, we can calculate the probability of failure of a service vessel versus time for the design parameters and degraded parameters, and also do the same for the fleet leaders. Thus in Figure 2 we plot

$$
F_{4, d}\left(t ; S_{o p}\right)=1-\exp \left\{-4(0.548)^{30}(t)^{0.25}\right\}
$$




$$
\begin{aligned}
& F_{4, D}\left(t ; S_{o p}\right)=1-\exp \left\{-4(0.548)^{18}(t)^{0.225}\right\} \\
& F_{10, d}\left(t ; S_{f l}\right)=1-\exp \left\{-10(0.789)^{30}(t)^{0.25}\right\}
\end{aligned}
$$

and

$$
F_{10, D}\left(t ; S_{f l}\right)=1-\exp \left\{-10(0.789)^{18}(t)^{0.225}\right\}
$$

Clearly evident in Figure 2 are the high probabilities of both a false alarm and failure to sound a true alarm.

\section{Case 3: Design of Fleet Leaders for T1000G Fiber COPVs for Constellation Program.}

In this example we consider a hypothetical fleet leader program design for Constellation Program. Let us say the typical vehicle is to carry $20 \mathrm{COPVs}$ at a burst factor (design burst pressure/maximum operating pressure) of about 2.0. This translates approximately to a stress ratio of 50 percent. The properties used are for a typical T1000G fiber overwrap. The parameters chosen for the model are $\rho_{d}=$ $72, t_{\mathrm{ref}, d}=0.001 \mathrm{hr}, \beta_{d}=0.35$. These are expected values of parameters for a flight size T1000G overwrapped pressure vessel based on very limited data that is available in literature. The model parameters chosen to represent pressure vessels with potential errors in design, materials quality control or manufacturing are $\rho_{D}=50, t_{\text {ref }, D}=0.001 \mathrm{hr}, \beta_{D}=0.20$. The reduced parameters would lead to a much lower reliability than acceptable levels by the strict program requirements. Number of fleet leaders needed and the operating conditions for the fleet leaders are considered as design parameters and are estimated based on the models developed in the current work.



Figure 2.-Operating curves for a carbon COPV Fleet Leader Program to guard against possible design/manufacturing flaws and poor material quality. 


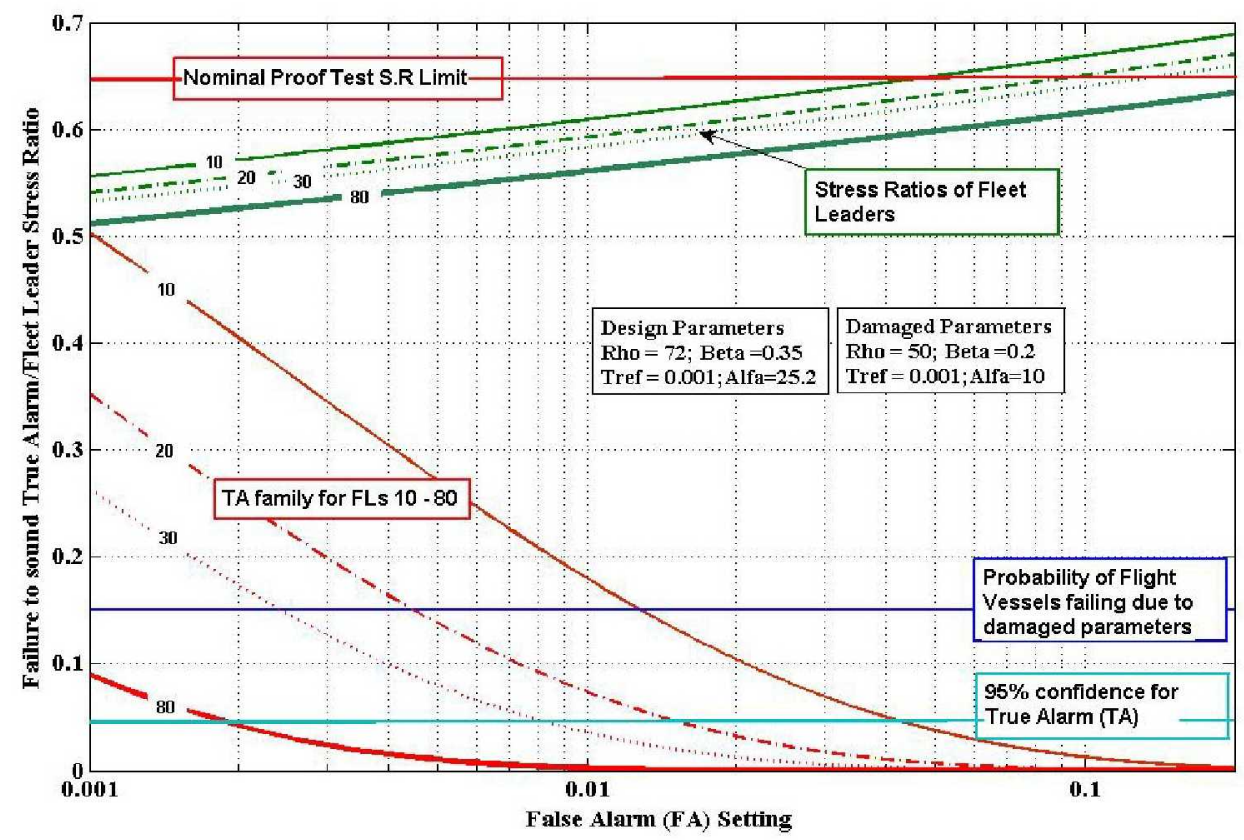

Figure 3.-Fleet leader design space for carbon Composite Overwrapped Pressure Vessels.

A family set of $10,20, \ldots ., 80$ fleet leaders are considered to arrive at the desired settings for the "false alarm" and "true alarm" as described below. The results are plotted in Figure 3. The horizontal axis in the figure represents a 'false alarm', meaning the chance of a fleet leader failure due to natural anticipated causes by the model. Both "failure to sound a true alarm" and "stress ratio" are plotted on the vertical axis. Here "failure to sound a true alarm" means that despite high risk from processing errors the fleet leaders have not sounded the alarm. As shown in the figure, under the assumed operating conditions (stress ratio $=50$ percent) and design life of 1 year $(8760 \mathrm{hr})$ of continuous operation, the probability of one of the 20 flight vessels failing due to degraded parameters of the model (to represent deficient or damaged vessels during manufacturing) is about 0.16 . Also plotted in the same figure are the fleet leader stress ratios as a function of "false alarm". For a "false alarm" setting of 0.02 (chance of natural fleet leader failure), it can be seen from the figure that we need at least 20 or more vessels in the fleet leader program to make sure that a true alarm sounds with 95 percent certainty. The stress ratios needed for the fleet leaders in order to keep the false alarm setting at 0.02 is around 60 percent which is significantly higher than the operating stress ratios in the fleet. False alarms cause costly program and schedule disruptions. In order to minimize such disruptions it is always desirable to reduce this setting to a lower value. For example, if we set false alarm to 0.01 (which corresponds to about 0.55 percent stress ratio) then from the figure we can see that the number of fleet leaders have to be increased to 25 or more so that the failure to sound a true alarm is less than 5 percent. The costs of schedule disruptions must be carefully weighed against the cost of keeping more fleet leaders in the program to set the optimum settings for the false alarm. The present methodology provides the necessary mathematical framework to perform such trade studies before setting up a fleet leader program.

\section{Fleet Leader Issues: Carbon vs. Kevlar}

Based upon the above results for the three different cases for Kevlar and carbon COPVs we can summarize the following critical issues for setting up fleet leader programs.

(1) One difficulty in the case of carbon COPVs is that the probabilities do not change very rapidly with time. The probabilities of a false alarm or true alarm are both high in the first few hours but the 
growth rate rapidly slows. This is because the Weibull lifetime shape parameter values are very low $\left(\beta_{d}=\right.$ 0.25 and $\beta_{D}=0.225$ ), and thus, there is a strongly decreasing hazard rate with time. The case with Kevlar COPVs was very different. In that case $\left(\beta_{d}=1.67\right.$ and $\left.\beta_{D}=2.5\right)$ so the hazard rate was strongly increasing.

(2) Another difficulty is the major difference in sensitivity in the two cases with respect to false alarms and true alarms. In the two Kevlar COPV cases, the probabilities of either giving a false alarm or failing to sound a true alarm were both no more than $1 / 67$ in the first example and about $1 / 200$ in the second example. In the carbon COPV case these probabilities were both much higher at about $1 / 7$. For carbon COPVs, this lack of discriminating ability lowers the usefulness of the fleet leaders. Unfortunately the scenario cited is typical of what is seen in carbon/epoxy strand data where $\rho$ values and $\alpha$ values can vary widely among data sets and between vessels and strands for the same fiber. Changes of fiber or matrix lead to large changes in model parameters.

(3) In the case of carbon COPVs, the stress ratios in the fleet leaders must be very high in order to obtain reasonably balanced sensitivities for false and true alarms when both operate in the same real time. In the above example, although a moderate design stress ratio, $S_{o p}=0.548$, is applied to the service vessels, the fleet leader stress ratio, $S_{f l}=0.7226$, despite being much higher, was poor at sounding a true alarm, when the vessel parameters reflected serious degradation or damage. The fiber stress ratio had to be raised to $S_{f l}=0.789$ to balance the sensitivities to avoiding false alarms and sounding true alarms.

(4) Difficulties arise also when the fleet leaders are not truly identical to the service vessels and there is uncertainty in exactly what the differences are. For instance in the carbon COPV case the fiber tress ratio that was needed, $S_{f l}=0.789$, is actually well above most proof test levels, typically 0.65 to 0.75 . While we have not considered proof testing in the above examples, the circumstances of the fleet leaders and service vessels are immediately very different since 'de facto' they have different proof levels. Unfortunately, proof testing by itself can introduce damage in terms of broken strands especially when above 0.70 . This becomes a critical parameter in the case of the fiber breakage reliability model (Refs. 2 and 13), which is more appropriate for carbon COPVs. Thus the relationship between the fleet leaders and service vessels becomes more uncertain, which adds to the difficulty of evaluating sensitivities in terms of true and false alarms.

(5) Finally, pure fleet leaders - i.e., vessels that are identical to service vessels in design, load and environment except service vessels accumulate time at risk much more slowly-require less sophistication in terms of a model than do fleet leaders that employ some form of acceleration. Despite the longstanding scientific basis for pressure and temperature acceleration, particularly the latter using Arrhenius laws, skepticism of engineers lacking sufficient foundation in probability and statistics as well as the stress-rupture phenomenon itself can cloud proper interpretation of true alarm versus false alarm sensitivities, when failures occur. If the underlying basis of these sensitivities is poorly understood, and the number of fleet leader vessels insufficient, then the benefit of a fleet leader program is open to serious question. The danger is that the observations over time - whether fleet leader failures occur or not - are likely to be incorrectly interpreted and may be rationalized or even manipulated in decision making. The risk is that truly degraded service vessels will end up being kept in service.

\section{Conclusions}

Stress rupture of composite overwraps can cause catastrophic consequences leading to loss of crew and spacecraft and hence the reliability of these vessels during the entire duration of a space program must be carefully examined and assessed. The present paper illustrates, via a classic stress rupture lifetime model (Refs. 1 and 6 to 10), how one can statistically design a "fleet leader" program, including fiber stress ratios and numbers of test vessels, as a risk mitigation step to supplement the original reliability 
calculations by analysis only, but with parameter values estimated using an available data base of similar material systems. Furthermore, the considerable complications arising in the case of carbon COPVs, due to widely differing model parameters for carbon versus Kevlar vessels, have been illustrated via specific examples.

\section{References}

1. Orbiter Kevlar/Epoxy Composite Overwrapped Pressure Vessel Flight Rationale Technical Assessment Report, vols. I and II, NASA NESC Report RP-07-34, April 12, 2007.

2. Shelf Life Phenomenon and Stress Rupture Life of Carbon/Epoxy Composite Overwrapped Pressure Vessels (COPVs) Technical Consultation Report, Vols. I and II, NASA NESC Report RP-06-83, September 14, 2006.

3. Glaser, R.E., R.L. Moore, and T.T. Chiao, "Life Estimation of an S-Glass/Epoxy Composite under Tensile Loading," Composites Technical Review, vol. 5, no. 1 (1983): 21-26.

4. Glaser, R.E., R.L. Moore, and T.T. Chiao, "Life Estimation of Aramid/Epoxy Composites under Sustained Tension," Composites Technical Review, vol. 26 (1984): 26.

5. Wu, H.F., S.L. Phoenix, and P. Schwartz, "Temperature Dependence of Lifetime Statistics for Single Kevlar 49 Filaments in Creep-Rupture," Journal of Materials Science, 23 (1988):1851.

6. Phoenix, S.L., and E.M. Wu, "Statistics for the Time Dependent Failure of Kevlar-49/Epoxy Composites: Micromechanical Modeling and Data Interpretation," IUTAM Symposium on Mechanics of Composite Materials, Pergamon (1983):135.

7. Coleman, B.D, "Statistics and Time Dependence of Mechanical Breakdown in Fibers," Journal of Applied Physics, 29 (1958): 968.

8. Phoenix, S.L, "Statistical Modeling of the Time and Temperature Dependent Failure of Fibrous Composites," Proceedings of the 9th US National Congress of Applied Mechanics, Book no. H00228, ASME, NY (1982): 219.

9. Phoenix, S.L., "The Asymptotic Time to Failure of a Mechanical System of Parallel Members," SIAM Journal on Applied Mathematics, 34 (1978): 227.

10. Phoenix, S.L., "Stochastic Strength and Fatigue of Fiber Bundles," International Journal of Fracture, 14 (1978): 327.

11. Helton J.C. and D.E. Burmaster, "Treatment of Aleatory and Epistemic Uncertainty in Performance Assessments for Complex Systems," Reliability Engineering and System Safety: Special Issue on Aleatory and Epistemic Uncertainties, 54 (1996): 91.

12. Hoffman F.O. and J.S. Hammonds, J.S., "Propagation of Uncertainty in Risk Assessments: The Need to Distinguish Between Uncertainty Due to Lack of Knowledge and Uncertainty Due to Variability," Reliability Engineering and System Safety, 14 (1994): 707.

13. Phoenix, S.L. and P.L.N. Murthy, "Pros and Cons of Proof Testing Carbon Composite Overwrapped Pressure Vessels: A Comparison of Two Mathematical Models," Proceedings of 48th AIAA/ASME/ASCE/AHS/ASC Structures, Structural Dynamics, and Materials Conference, April 23, 2007, Honolulu, Hawaii. 


\begin{tabular}{|c|c|c|}
\hline \multicolumn{2}{|c|}{ REPORT DOCUMENTATION PAGE } & $\begin{array}{c}\text { Form Approved } \\
\text { OMB No. 0704-0188 }\end{array}$ \\
\hline \multicolumn{3}{|c|}{$\begin{array}{l}\text { The public reporting burden for this collection of information is estimated to average } 1 \text { hour per response, including the time for reviewing instructions, searching existing data sources, gathering and maintaining the } \\
\text { data needed, and completing and reviewing the collection of information. Send comments regarding this burden estimate or any other aspect of this collection of information, including suggestions for reducing this } \\
\text { burden, to Department of Defense, Washington Headquarters Services, Directorate for Information Operations and Reports (00704-0188), 1215 Jefferson Davis Highway, Suite } 1204 \text {, Arlington, VA 22202-4302. } \\
\text { Respondents should be aware that notwithstanding any other provision of law, no person shall be subject to any penalty for failing to comply with a collection of information if it does not display a currently valid OMB } \\
\text { control number. } \\
\text { PLEASE DO NOT RETURN YOUR FORM TO THE ABOVE ADDRESS. }\end{array}$} \\
\hline $\begin{array}{l}\text { 1. REPORT DATE (DD-MM-YYYY) } \\
01-10-2009\end{array}$ & $\begin{array}{l}\text { 2. REPORT TYPE } \\
\text { Technical Memorandum }\end{array}$ & 3. DATES COVERED (From - To) \\
\hline \multirow{3}{*}{\multicolumn{2}{|c|}{$\begin{array}{l}\text { 4. TITLE AND SUBTITLE } \\
\text { Designing of a Fleet-Leader Program for Carbon Composite Overw }\end{array}$}} & 5a. CONTRACT NUMBER \\
\hline & & 5b. GRANT NUMBER \\
\hline & & 5c. PROGRAM ELEMENT NUMBER \\
\hline \multirow{3}{*}{\multicolumn{2}{|c|}{$\begin{array}{l}\text { 6. AUTHOR(S) } \\
\text { Murthy, Pappu, L.N.; Phoenix, S., Leigh }\end{array}$}} & 5d. PROJECT NUMBER \\
\hline & & 5e. TASK NUMBER \\
\hline & & $\begin{array}{l}\text { 5f. WORK UNIT NUMBER } \\
\text { WBS 869021.03.03.02.01 }\end{array}$ \\
\hline \multicolumn{2}{|c|}{$\begin{array}{l}\text { 7. PERFORMING ORGANIZATION NAME(S) AND ADDRESS(ES) } \\
\text { National Aeronautics and Space Administration } \\
\text { John H. Glenn Research Center at Lewis Field } \\
\text { Cleveland, Ohio 44135-3191 }\end{array}$} & $\begin{array}{l}\text { 8. PERFORMING ORGANIZATION } \\
\text { REPORT NUMBER } \\
\text { E-17057 }\end{array}$ \\
\hline \multirow{2}{*}{\multicolumn{2}{|c|}{$\begin{array}{l}\text { 9. SPONSORING/MONITORING AGENCY NAME(S) AND ADDRESS(ES) } \\
\text { National Aeronautics and Space Administration } \\
\text { Washington, DC 20546-0001 }\end{array}$}} & $\begin{array}{l}\text { 10. SPONSORING/MONITOR'S } \\
\text { ACRONYM(S) } \\
\text { NASA; AIAA }\end{array}$ \\
\hline & & $\begin{array}{l}\text { 11. SPONSORING/MONITORING } \\
\text { REPORT NUMBER } \\
\text { NASA/TM-2009-215685; AIAA-2009- } \\
2517\end{array}$ \\
\hline \multicolumn{3}{|c|}{$\begin{array}{l}\text { 12. DISTRIBUTION/AVAILABILITY STATEMENT } \\
\text { Unclassified-Unlimited } \\
\text { Subject Category: } 24 \\
\text { Available electronically at http://gltrs.grc.nasa.gov } \\
\text { This publication is available from the NASA Center for AeroSpace Information, 443-757-5802 }\end{array}$} \\
\hline
\end{tabular}

\section{SUPPLEMENTARY NOTES}

\section{ABSTRACT}

Composite Overwrapped Pressure Vessels (COPVs) are often used for storing pressurant gases on board spacecraft when mass saving is a prime requirement. Substantial weight savings can be achieved compared to all metallic pressure vessels. For example, on the space shuttle, replacement of all metallic pressure vessels with Kevlar COPVs resulted in a weight savings of about 30 percent. Mass critical space applications such as the Ares and Orion vehicles are currently being planned to use as many COPVs as possible in place of all-metallic pressure vessels to minimize the overall mass of the vehicle. Due to the fact that overwraps are subjected to sustained loads during long periods of a mission, stress rupture failure is a major concern. It is, therefore, important to ascertain the reliability of these vessels by analysis, since it is practically impossible to show by experimental testing the reliability of flight quality vessels. Also, it is a common practice to set aside flight quality vessels as "fleet leaders" in a test program where these vessels are subjected to slightly accelerated operating conditions so that they lead the actual flight vessels both in time and load. The intention of fleet leaders is to provide advanced warning if there is a serious design flaw in the vessels so that a major disaster in the flight vessels can be averted with advance warning. On the other hand, the accelerating conditions must be not so severe as to be prone to false alarms. The primary focus of the present paper is to provide an analytical basis for designing a viable fleet leader program for carbon COPVs. The analysis is based on a stress rupture behavior model incorporating Weibull statistics and power-law sensitivity of life to fiber stress level.

\section{SUBJECT TERMS}

Composite overwrapped pressure vessels; Stress rupture; Weibull statistics; Stress rupture life; Liner loadsharing; Burst pressure; Operating pressure; Fiber strength; Pressurization rate; Power law; Confidence intervals; Reliability statistics

\begin{tabular}{|c|c|c|c|c|c|}
\hline \multicolumn{3}{|c|}{ 16. SECURITY CLASSIFICATION OF: } & \multirow{2}{*}{$\begin{array}{l}\text { 17. LIMITATION OF } \\
\text { ABSTRACT } \\
\text { UU }\end{array}$} & \multirow{2}{*}{$\begin{array}{l}\text { 18. NUMBER } \\
\text { OF } \\
\text { PAGES } \\
23\end{array}$} & \multirow{2}{*}{$\begin{array}{l}\text { 19a. NAME OF RESPONSIBLE PERSON } \\
\text { STT Help Desk (email:help@sti.nasa.gov) } \\
\text { 19b. TELEPHONE NUMBER (include area code) } \\
\text { 443-757-5802 }\end{array}$} \\
\hline $\begin{array}{l}\text { a. REPORT } \\
\mathrm{U}\end{array}$ & $\begin{array}{l}\text { b. ABSTRACT } \\
\mathrm{U}\end{array}$ & $\begin{array}{l}\text { c. THIS } \\
\text { PAGE } \\
\text { U }\end{array}$ & & & \\
\hline
\end{tabular}



